Original article

\title{
Nuclear factor of activated T (NFAT) cells activity within CD4+ T cells is influenced by activation status and tissue localisation
}

\author{
Nicola L. Harris ${ }^{\mathrm{a}, *}$, Victoria Watt ${ }^{\mathrm{a}}$, Sam MacLenachan ${ }^{\mathrm{a}}$, Sean Diehl ${ }^{\mathrm{b}}$, Benjamin J. Marsland ${ }^{\mathrm{a}}$, \\ Mercedes Rincon ${ }^{\mathrm{b}}$, Graham Le Gros ${ }^{\mathrm{a}}$ \\ ${ }^{a}$ Malaghan Institute of Medical Research, Central Services Building, Victoria University, Wellington, New Zealand \\ ${ }^{\mathrm{b}}$ Department of Medicine/Immunobiology Program, Given Medical Building D305, University of Vermont, Burlington, VT 05405, USA
}

Received 17 March 2005; accepted 17 June 2005

Available online 12 September 2005

\begin{abstract}
Nuclear factor of activated T (NFAT) cells is a family of transcription factors important for the regulation of cytokine expression by CD4+ T cells. Whilst a number of studies have examined NFAT activity of in vitro generated CD4+ T helper (Th) 1 and Th2 cells, regulation of NFAT during in vivo immune responses has yet to be elucidated. We show that NFAT activity in CD4+ T cells peaked at early time-points in the draining mediastinal lymph node of mice infected with influenza A (Flu) or Nippostrongylus brasiliensis (Nb). In contrast, low NFAT transcriptional activity was detected in CD4+ T cells isolated from the lung of either Flu or Nb infected mice, despite a greater proportion of cytokine-producing cells being present at this site. These findings indicate that the activation status and tissue microenvironment of effector CD4+ T cells can determine their requirement for NFAT-mediated transcription.
\end{abstract}

(C) 2005 Elsevier SAS. All rights reserved.

Keywords: CD4+ T cell; Lymph node; Lung; Influenza; Parasite

\section{Introduction}

Although nuclear factor of activated T (NFAT) was originally described as a transcription factor present in the nucleus of activated T cells, it is now clear that NFAT represents a family of transcription factors which are expressed in other cells of the lymphoid system as well as non-lymphoid cells (reviewed by [1,2]). This family of proteins is characterised by the presence of a Rel-homology domain and a calcineurin binding domain. Four members of the family have been identified, NFATc1, NFATc2, NFATc3 and NFATc4. NFATc 1 and NFATc 2 are the predominant members in T cells, but they are also expressed in a large variety of tissues including heart, neurons, vascular cells, chondriocytes, and adipocytes [3-7].

NFAT transcription factors are believed to be present in the cytoplasm and translocated to the nucleus in response to calcium signals [8]. The accumulation of NFAT in the nucleus

\footnotetext{
* Corresponding author. Present address: Environmental Biomedicine, ETHZ, Wagistrasse 25/27, 8952 Schlieren. Tel.: +41 44633 6790; fax: +41446331582.

E-mail address: nharris@ethz.ch (N.L. Harris).
}

is the result of nuclear import promoted by calcineurinmediated dephosphorylation, and nuclear export promoted by phosphorylation mediated by several kinases (e.g. GSK3, JNK, p38 MAP kinase, casein-kinase II, MKK1) [9-14]. In the nucleus, NFAT family members associate with other nuclear transcription factors (e.g. JUN, GATA4, IRF, C/EBP) depending on the cell type, to mediate transcription of specific genes including cytokine and cytokine receptor genes [15-17]. In addition to the regulation of their nuclear localisation by phosphorylation/dephosphorylation, NFAT members can also be regulated at the level of gene expression. The expression of NFATc1 isoforms is regulated during the activation of CD4+ $\mathrm{T}$ cells by the usage of different promoters and corresponding polyadenylation signals [18-20]. NFATc2 expression is also upregulated during the activation of CD4+ T cells and by IL-6-mediated signals [21].

In addition to nuclear transport, phosphorylation can also regulate NFAT activity in a different manner. Although less well established, there are some studies indicating that phosphorylation can regulate the ability of NFAT members to mediate transcription once they are in the nucleus. PIM-1mediated phosphorylation has been shown to be important 
for the transactivation activity of NFATc1 [22]. The mechanism remains unclear, but it is possible that phosphorylation can regulate the association of NFAT to other transcription factors or co-activators. Thus, several regulatory mechanisms control NFAT activity.

A number of studies have shown the regulation of NFAT nuclear accumulation and transcription during activation of naïve CD4+ T cells and differentiation of these cells into effector Th1 and Th2 CD4+ T cells in vitro. It has been shown that NFAT transcriptional activity is rapidly induced to higher levels in effector cells compared to naïve CD4+ T cells following activation [23]. Whilst no difference in nuclear accumulation of NFAT has been observed during the differentiation of Th1 and Th2 cells [24], higher NFAT transcriptional activity is triggered in Th2 cells compared with that triggered in Th1 cells in vitro [23]. To date, no studies have tried to address the regulation of NFAT activity in effector CD4+ T cells during in vivo Th1 and Th2 immune responses.

In the present study, we have examined the regulation of NFAT activity during the activation of effector T helper cells generated in vivo in response to influenza virus (Th1) or Nippostrongylus brasiliensis (Th2). NFAT transcriptional activity was found at maximal levels within CD4+ T cells isolated from the draining mediastinal lymph node (MedLN) of infected mice, yet activity was decreased as the ability to produce effector cytokines was gained. NFAT activity in T cells isolated from the lung was consistently reduced in comparison to levels observed in MedLN cells. This was in direct contrast to the increased capacity of these cells for production of the effector cytokines IFN- $\gamma$ and IL-4. These data were supported by analysis of NFAT DNA binding activity by EMSA in which NFAT activity was detected in both LN and lung samples, but was consistently decreased in those cells isolated from the lung.

\section{Materials and methods}

\subsection{Mice}

All mice were bred and maintained at the Animal Facility of the Wellington School of Medicine. NFAT-luciferase reporter transgenic mice have been ${ }^{1}$ described previously [23]. These mice were backcrossed at least six times onto the C57BL/6 background, and maintained by breeding female transgenic mice to male C57BL/6 mice. All experimental animal procedures used in this study were approved by the Wellington School of Medicine Animal Ethics Committee, and carried out in accordance with the guidelines set by the University of Otago, New Zealand.

\subsection{Influenza A and N. brasiliensis infections}

The A/HKx31 (H3N2) influenza A virus was maintained as described [25]. For Flu infection, mice were anaesthetised by intraperitoneal injection (i.p.) of a mixture of ketamine and xylazine (Phoenix, Auckland, New Zealand) and 12 HAU of virus in a $30 \mu \mathrm{l}$ volume of PBS was administered by intranasal inoculation (i.n.). The preparation of $\mathrm{Nb}$ third stage larvae (L3) and the subcutaneous inoculation of L3 were performed as described previously [26]. Mice were infected with 750 L3, then subjected to a second infection of 1000 L3 8 weeks later.

\subsection{CD4+ T cell isolation}

For the preparation of mononuclear cells from the lung, mice were sacrificed and the lungs perfused via the heart right ventricle with approximately $5 \mathrm{ml}$ PBS to remove circulating blood. Minced lung tissue was incubated for $1 \mathrm{~h}$ in complete IMDM containing $2.4 \mathrm{mg} / \mathrm{ml}$ collagenase type II (GibcoBRL) and $0.1 \%$ DNase 1 (Sigma), and mononuclear cells purified by gradient centrifugation over $30 \%$ Percoll@. Complete IMDM (cIMDM) consisted of IMDM (Sigma, Chemical Co., St. Louis, MO, USA) with 5\% FCS (Gibco BRL, Life Tech, Auckland, New Zealand), $2 \mathrm{mM}$ glutamine (Sigma), $1 \%$ penicillin-streptomycin (Sigma) and $5 \times 10^{-}$ 5 M 2-ME (Sigma). MedLN cell suspensions were prepared by squeezing the lymph nodes between two layers of gauze. For all samples CD4+ T cells were isolated by labelling with biotin labelled anti-CD4 mAb (GK1.5), followed by purification using Strepdavidin MicroBeads ${ }^{\circledR}$ (MACs Reagents, Miltenyi Biotec, Germany) as per manufacturer's instructions. Purity of CD4+ T cells was $80-95 \%$.

\subsection{Measurements of luciferase content}

CD4+ T cells purified from infected or non-infected mice were re-stimulated on anti-CD3 mAb $(2 \mathrm{C} 11,10 \mathrm{~g} / \mathrm{ml})$ coated 24 well plates in supplemented IMDM plus $20 \mathrm{U} / \mathrm{ml}$ recombinant human IL-2 and anti-CD28 mAb for $20 \mathrm{~h}$. Harvested cells were counted and samples adjusted to contain between $5 \times 10^{5}$ and $1 \times 10^{6}$ live cells. Cells were washed twice in PBS then lysed in lysis buffer (Luciferase Assay, Promega, Madison, WI) for $30 \mathrm{~min}$ at RT. Lysate was centrifuged and supernatant analysed using Luciferase Reagent (Promega). Measurements were made for $10 \mathrm{~s}$ using a luminometer (TD 20/20, Turner designs, Sunnyvale, CA). Three measurements were made of each sample, with background readings of $\leq 0.05$. Luciferase values were averaged, corrected to represent measurements for $1 \times 10^{6}$ cells, then expressed as the fold increase in activity determined for CD4+ T cells isolated from infected versus non-infected mice.

\subsection{Intracellular cytokine staining and FACs analysis}

CD4+ T cells purified from the lung or MedLN were cultured at a concentration of $1 \times 10^{6}$ cells per well in 24 well plates (Falcon) for a total of $20 \mathrm{~h}$ as indicated. All cells were incubated with or without plate bound anti-CD3 $\mathrm{mAb}$ in a total volume of $1 \mathrm{ml}$ of cIMDM plus IL-2 and antiCD28 mAb. Monensin (Calbiochem-Novabiochem Corp., La 
Jolla, CA, USA) was added at a final concentration of $2 \mathrm{mM}$ for the last 3-4 h of culture. At the end of the culture period cells were harvested, washed once in PBS buffer, then fixed in $4 \%$ paraformaldehyde (Sigma) for $15 \mathrm{~min}$ at RT. Fixed cells were surface stained with anti-CD4-Biotin mAb followed by SA-PerCP (Pharmingen) then subjected to intracellular cytokine staining in saponin buffer (PBS, $10 \mathrm{mM}$ Hepes, $0.1 \%$ saponin, $0.1 \%$ BSA, $0.02 \% \mathrm{Na}$ azide) for $30 \mathrm{~min}$ at RT. Anti-IL-4-APC and anti-IFN- $\gamma$-PE were obtained from Pharmingen and used at a 1:100 dilution. Flow cytometric analysis was performed on a FACSort (Becton Dickinson) using the CellQuest software.

\subsection{Nuclear extracts and electrophoretic mobility shift assay (EMSA)}

CD4+ T cells were isolated from mice at day 4 following infection then stimulated on anti-CD3 coated plates in the presence of anti-CD28 and IL-2 for $20 \mathrm{~h}$. At the end of culture period nuclear proteins were extracted as previously described [27]. Binding reactions were carried out using $1.5 \mu \mathrm{g}$ of nuclear proteins and a 32P-dCTP-end labelled double stranded oligonucleotide probe containing a NFAT binding site from the proximal IL-4 promoter 5 '-GTA ATA AAA TTT TCC AAT GTA AA-3' [28] or a CREB binding site from the somatostatin gene 5'-GAT CTC TCT GAC AGC CAA GGA GGA GGC C-3' [29]. One millilitre of anti-NFATc1 Ab (Upsate Biotechnology, Lake Placid, NY), or anti-NFATc2 $\mathrm{mAb}$ (Affinity BioReagents, Golder, $\mathrm{CO}$ ), was present in the binding reactions for super-shift analysis. Samples were electrophoresed under non-denaturing conditions and exposed to film for autoradiography.

\section{Results}

\subsection{NFAT-driven transcription is increased in CD4+ $T$ cells early following influenza A virus infection, but decreases as effector function is gained}

Flu infects the epithelial cells lining the airways [30], and in mice induces a strong immune response typically resulting in the eradication of virus by day 10 post-infection (unpublished data from our laboratory). Protective immunity is largely due to the action of neutralising antibodies and cytolytic CD8+ T cells, but is paralleled by the accumulation of effector Th1 cells in the MedLN and lung $[31,32]$. IFN- $\gamma$ production plays an important role in anti-viral immunity as demonstrated by a dramatically delayed clearance of the virus in mice treated with anti-IFN- $\gamma$ antibodies $[33,34]$.

Infection of C57BL/6 mice with Flu led to an increased number of CD4+ T cells present in the MedLN from day 4, and the lung from day 8 (Fig. 1A). These cells developed the capacity to produce IFN- $\gamma$ by day 8 , with increased numbers of IFN- $\gamma$-producing CD4+ T cells detected at day 12 postinfection (Fig. 1B). The proportion of effector CD4+ Th1 cells

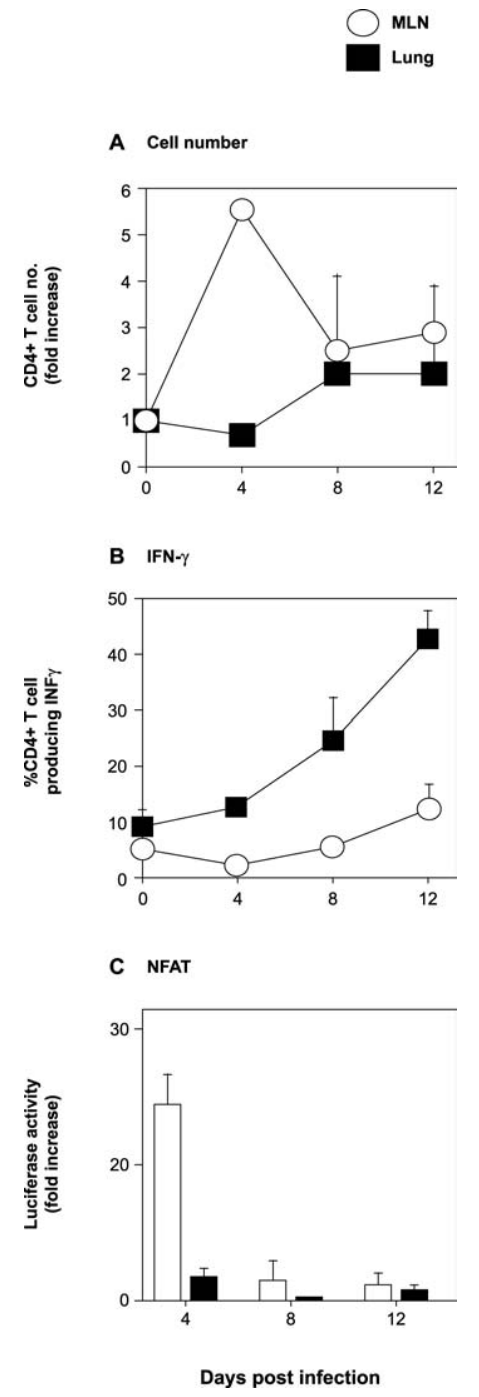

Fig. 1. NFAT transcriptional activity in $\mathrm{CD} 4+\mathrm{T}$ cells isolated from the MedLN or lung of influenza A infected mice. C57BL/6 (A and B) or NFATluciferase reporter transgenic mice $(\mathbf{C})$ were given an i.n. inoculation $12 \mathrm{HAU}$ of influenza A virus in a $30 \mu \mathrm{l}$ volume. (A) At the indicated time-points following infection, lymphocyte suspensions from the MedLN or lung were counted and examined for CD4 expression. Plots show fold increase in the total numbers of CD4+ T cells in infected versus naïve mice. (B) Remaining cells were re-stimulated on plate bound anti-CD3 for $20 \mathrm{~h}$, with monensin added for the final 3-4 h of culture. Cells were then fixed and subjected to intracellular cytokine staining as described in Section 2. Plots indicate the proportion of CD4+ T cells producing IFN- $\gamma$. (C) $\mathrm{CD} 4+\mathrm{T}$ cells isolated from MedLN or lung of naïve or Flu infected NFAT-luciferase reporter transgenic mice were re-stimulated on plate bound anti-CD3 for $20 \mathrm{~h}$, and luciferase activity determined as described. Values indicate fold increase in absolute luminometer readings per $1 \times 10^{6}$ cells isolated from infected versus naïve mice. The data shown represents mean \pm S.D. of two to four individual animals from one representative experiment. Similar results were obtained from three separate experiments.

(as determined by IFN- $\gamma$ production) present in the lung was found to increase from day 8 and was much greater than that observed in the draining MedLN (Fig. 1B).

We sought to analyse the utilisation of NFAT by CD4+ T cells following Flu infection. For this purpose we employed the use of transgenic mice in which NFAT-driven transcription can be determined by the expression of a luciferase 
reporter gene. These mice have been used extensively for analysis of NFAT-driven transcriptional activity in different tissues $[21,23,35,36]$. For detection of luciferase expression, CD4+ T cells obtained from naïve or Flu infected mice were re-stimulated in vitro for $20 \mathrm{~h}$ with anti-CD3 mAb. A greater than 20-fold increase in NFAT transcriptional activity was detected in CD4+ T cells isolated from the MedLN at day 4 after Flu infection compared to naïve control mice (Fig. 1C). However, this increase was short-lived, and no longer apparent by day 8 post-infection (Fig. 1C). No NFAT activity could be detected in lung CD4+ T cells at any time-point examined (Fig. 1C), despite large numbers of these cells being capable of producing IFN- $\gamma$ (Fig. 1B). The lack of T cell luciferase expression in these assays was not a consequence of poor activation as the culture supernatant was found to contain increased levels of IFN- $\gamma$ indicating normal effector function (data not shown).

\subsection{Increased NFAT-mediated transcription in lymph node} resident effector $C D 4+T$ cells compared to lung resident cells following $N$. brasiliensis infection

We next examined NFAT-driven transcriptional activity in the MedLN and lung of $\mathrm{Nb}$ infected mice. $\mathrm{Nb}$ is a helminth parasite that penetrates its host via the skin, then migrates into the vasculature, becoming trapped within the lungs by 24-48 h post-infection. Larvae are coughed up and swallowed 48-72 $\mathrm{h}$ after inoculation and mature in the jejunum into egg-laying adults by days 5-6 [37]. Mice are protected against a challenge infection with $\mathrm{Nb}$ [38], however some worms do make it to the lung and challenge infection results in a rapid and marked Th2 cell response at this site, which is greater than that seen following primary infection (unpublished observations) and characterised by IL-4 and IL-5 production and eosinophilia. We therefore used a challenge infection with $\mathrm{Nb}$ to examine IL-4 production in CD4+ T cells isolated from the MedLN or lung (Fig. 2A). Cells producing IL-4 could be detected by day 4, with cytokine-producing cell numbers peaking at day 6 in both tissues (Fig. 2B). As seen following Flu infection, the proportion of CD4+ T cells capable of producing effector cytokines (IL-4) was found to be much greater in the lung than the MedLN (Fig. 2B).

Analysis of the luciferase content of CD4+ T cells from $\mathrm{Nb}$ infected mice showed that NFAT transcriptional activity increased in CD4+ T cells isolated from the MedLN, with activity peaking at day 4 post-infection (Fig. 2C). Effector CD4+ T cells isolated from the lung only exhibited NFAT transcriptional activity at day 4 post-infection, and this was reduced in comparison to the activity detected in MedLN cells (Fig. 2C).

3.3. NFAT DNA binding activity is greater in MedLN than lung CD4+ Th1 and Th2 cells isolated from mice infected with influenza A virus or $N$. brasiliensis, respectively

In parallel we examined the NFAT DNA binding activity in $\mathrm{CD} 4+\mathrm{T}$ cells isolated from $\mathrm{Flu}$ or $\mathrm{Nb}$ infected

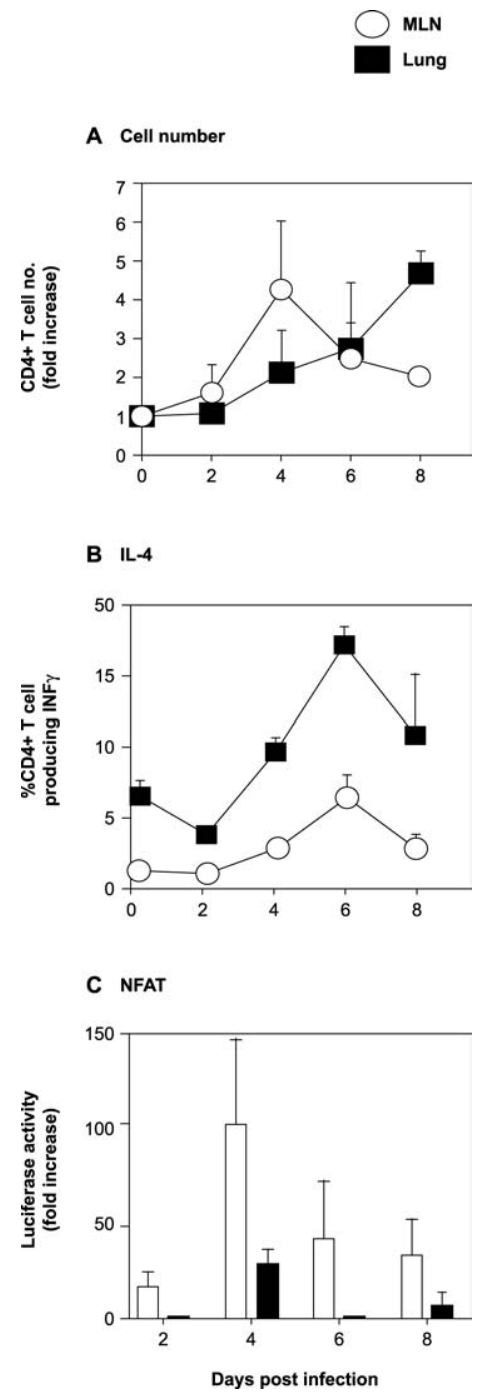

Fig. 2. NFAT transcriptional activity by CD4+ T cells isolated from the MedLN or lung of $N$. brasisliensis infected mice. C57BL/6 (A and B) or NFAT-luciferase reporter transgenic mice $(\mathbf{C})$ were given a primary s.c. inoculation with $750 \mathrm{Nb} \mathrm{L3}$, followed by a secondary s.c. inoculation of $1000 \mathrm{~L} 3$ 8 weeks later. At the indicated time-points following secondary infection the: (A) fold increase in total numbers of CD4+ T cells in infected versus naïve mice, (B) proportion of CD4+ T cells producing IL-4 and (C) fold increase in absolute luminometer readings per $1 \times 10^{6}$ cells isolated from infected versus naïve mice, were determined as described in Fig. 1. The data shown represents mean \pm S.D. of three to five individual animals from one representative experiment. Similar results were obtained from two separate experiments.

C57BL/6 mice. CD4+ T cells were isolated from the MedLN or lung at days 4-5 post-infection and re-stimulated on antiCD3 coated plates in the presence of anti-CD28 and IL-2 for $20 \mathrm{~h}$. At the end of culture period nuclear proteins were isolated and NFAT DNA binding activity was determined by EMSA. Protein loading was controlled by concurrent examination of CREB DNA binding activity. NFAT DNA binding activity was found to be present in nuclear extracts of both MedLN and lung CD4+ T cells isolated from mice infected with Flu (Fig. 3A) or Nb (Fig. 3B). However, in correlation with the results obtained for the regulation of NFAT-mediated 


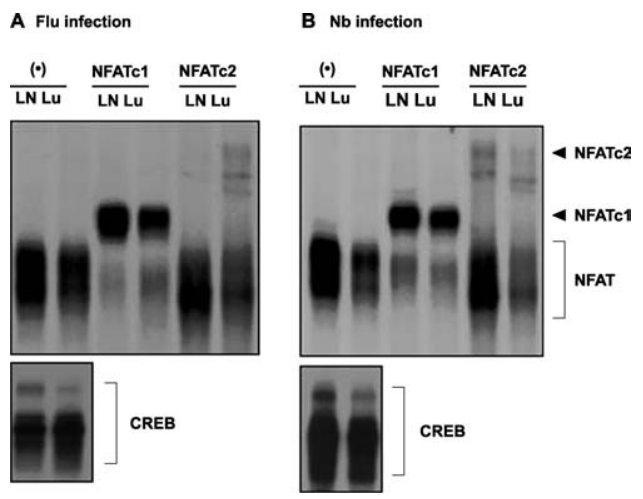

Fig. 3. Characterisation of NFAT members in CD4+ T cells isolated from the MedLN or lung of mice infected with influenza A or N. brasisliensis. CD4+ T cells were isolated from the MedLN and lung of (A) Flu or (B) Nb infected C57BL/6 mice and nuclear extracts made as described in Section 2. DNA binding activity of NFAT transcription factors were examined using an EMSA, and the characterisation of NFAT proteins as NFATc1 or NFATc2 determined by super-shift analysis using mAbs. DNA binding activity of CREB proteins is shown as a control for protein loading. The data shown are from nuclear extracts purified from pooled CD4+ T cells of 10-12 mice. Similar results were obtained from two separate experiments.

transcription, NFAT DNA binding was diminished in those CD4+ $\mathrm{T}$ cells isolated from the lung as compared to the MedLN (Fig. 3A, B). By performing supershift analysis we determined that this difference was apparent for both NFATc1 and NFATc2, which are the predominant NFAT family members present in T cells.

\section{Discussion}

We have observed that NFAT is transcriptionally active to a high degree in MedLN CD4+ T cells early following influenza or Nb infections, respectively. However, in both infection models, NFAT-driven transcription decreased as the cells become more activated. Moreover, effector CD4+ cells isolated from the lung of Flu infected mice did not express detectable luciferase levels, yet were capable of IFN- $\gamma$ production. In parallel NFAT-mediated transcription was decreased in effector CD4+ cells isolated from the lung compared to the draining $\mathrm{LN}$ of $\mathrm{Nb}$ infected mice.

Taken together, these data suggest that the activation of CD4+ T cells in the lymphoid tissues requires greater NFATmediated gene transcription, than does effector function of $\mathrm{T}$ cells residing in the lung. Thus, NFAT activity does not necessarily correlate with the degree of effector cytokine production. This may indicate that $\mathrm{T}$ cells require less NFATmediated transcription for cytokine production once they have undergone the process of chromatin remodelling and induction of cytokine gene transcription following initial encounter with antigen in the draining lymphoid tissues.

In a previous study we identified crucial differences in the ability of effector Th2 cells to proliferate within lymph node versus lung tissues following antigenic stimulation in vivo [39]. Our current observations indicate that, as for proliferation, NFAT-driven gene transcription is differentially regu- lated within lymphoid tissue and lung micro-environments. This supports our earlier hypothesis that effector CD4+ T cells are likely to receive varied signalling inputs when located within different organs. Such differences may arise through altered levels of antigen, co-stimulatory signals or through the presence of specific regulatory factors present within peripheral tissues.

In summary, we have observed that NFAT-mediated gene transcription was apparent at a greater level during the early stages of CD4+ $\mathrm{T}$ cell activation, and was active to a higher degree in $\mathrm{T}$ cells located within the draining lymphoid tissues compared to those cells present in the lung. These data highlight the important roles activation status and tissue microenvironment can play in the regulation of CD4+ T cell function, and have important implications for the use of NFAT inhibitors in treating pathological inflammation within peripheral tissues.

\section{Acknowledgments}

The authors thank the personnel of the animal facility of the Wellington School of Medicine for animal husbandry. This work was supported by grants from the Marsden Fund, Royal Society of New Zealand, Wellington Medical Research Foundation and the Wellcome Trust, UK.

\section{References}

[1] G.R. Crabtree, E.N. Olson, NFAT signalling: choreographing the social lives of cells, Cell (2002) 109 (Suppl.).

[2] A. Rao, C. Luo, P.G. Hogan, Transcription factors of the NFAT family: regulation and function, Annu. Rev. Immunol. 15 (1997) 707-747.

[3] J.L. de la Pompa, L.A. Timmerman, H. Takimoto, H. Yoshida, A.J. Elia, E. Samper, J. Potter, A. Wakeham, L. Marengere, B.L. Langille, G.R. Crabtree, T.W. Mak, Role of the NF-ATc transcription factor in morphogenesis of cardiac valves and septum, Nature 392 (1998) 182-186.

[4] I.A. Graef, F. Chen, L. Chen, A. Kuo, G.R. Crabtree, Signals transduced by $\mathrm{Ca}(2+) /$ calcineurin and NFATc3/c4 pattern the developing vasculature, Cell 105 (2001) 863-875.

[5] A.M. Ranger, M.J. Grusby, M.R. Hodge, E.M. Gravallese, F.C. de la Brousse, T. Hoey, C. Mickanin, H.S. Baldwin, L.H. Glimcher, The transcription factor NF-ATc is essential for cardiac valve formation, Nature 392 (1998) 186-190.

[6] A.M. Ranger, L.C. Gerstenfeld, J. Wang, T. Kon, H. Bae, E.M. Gravallese, M.J. Glimcher, L.H. Glimcher, The nuclear factor of activated T cells (NFAT) transcription factor NFATp (NFATc2) is a repressor of chondrogenesis, J. Exp. Med. 191 (2000) 9-22.

[7] I.C. Ho, J.H. Kim, J.W. Rooney, B.M. Spiegelman, L.H. Glimcher, A potential role for the nuclear factor of activated $\mathrm{T}$ cells family of transcriptional regulatory proteins in adipogenesis, Proc. Natl. Acad. Sci. USA 95 (1998) 15537-15541.

[8] N.A. Clipstone, G.R. Crabtree, Identification of calceniurin as a key signalling enzyme in T cell activation, Nature 357 (1992) 695-697.

[9] C.R. Beals, C.M. Sheridan, C.W. Turck, P. Gardner, G.R. Crabtree, Nuclear export of NF-ATc enhanced by glycogen synthase kinase-3, Science 275 (1997) 1930-1934.

[10] C.-H. Chow, M. Rincón, J. Cavanagh, M. Dickens, R.J. Davis, Nuclear accumulation of NFAT4 opposed by the JNK signal transduction pathway, Science 278 (1997) 1638-1641. 
[11] C.W. Chow, C. Dong, R.A. Flavell, R.J. Davis, c-Jun NH(2)-terminal kinase inhibits targeting of the protein phosphatase calcineurin to NFATc1, Mol. Cell. Biol. 20 (2000) 5227-5234.

[12] P. Gomez del Arco, S. Martinez-Martinez, J.L. Maldonado, I. OrtegaPerez, J.M. Redondo, A role for the p38 MAP kinase pathway in the nuclear shuttling of NFATp, J. Biol. Chem. 275 (2000) 13872-13878.

[13] T.T. Yang, Q. Xiong, H. Enslen, R.J. Davis, C.W. Chow, Phosphorylation of NFATc4 by p38 mitogen-activated protein kinases, Mol. Cell. Biol. 22 (2002) 3892-3904.

[14] J. Zhu, F. Shibasaki, R. Price, J.C. Guillemot, T. Yano, V. Dotsch, G. Wagner, P. Ferrara, F. McKeon, Intramolecular masking of nuclear import signal on NF-AT4 by casein kinase I and MEKK1, Cell 93 (1998) 851-861.

[15] J. Rengarajan, K.A. Mowen, K.D. McBride, E.D. Smith, H. Singh, L.H. Glimcher, Interferon regulatory factor 4 (IRF4) interacts with NFATc2 to modulate interleukin 4 gene expression, J. Exp. Med. 195 (2002) 1003-1012.

[16] F. Macian, C. Lopez-Rodriguez, A. Rao, Partners in transcription: NFAT and AP-1, Oncogene 20 (2001) 2476-2489.

[17] J.D. Molkentin, J.-R. Lu, C.L. Antos, B. Markham, J. Richardson, J. Robbins, S.R. Grant, E.N. Olson, A calcineurin-dependent transcriptional pathway for cardiac hypertrophy, Cell 93 (1998) 215-226.

[18] S. Chuvpilo, E. Jankevics, D. Tyrsin, A. Akimzhanov, D. Moroz, M.K. Jha, J. Schulze-Luehrmann, B. Santner-Nanan, E. Feoktistova, T. Konig, A. Avots, E. Schmitt, F. Berberich-Siebelt, A. Schimpl, E. Serfling, Autoregulation of NFATc1/A expression facilitates effector $\mathrm{T}$ cells to escape from rapid apoptosis, Immunity 16 (2002) 881-895.

[19] S. Chuvpilo, A. Avots, F. Berberich-Siebelt, J. Glockner, C. Fischer, A. Kerstan, C. Escher, I. Inashkina, F. Hlubek, E. Jankevics, T. Brabletz, E. Serfling, Multiple NF-ATc isoforms with individual transcriptional properties are synthesized in T lymphocytes, J. Immunol. 162 (1999) 7294-7301.

[20] S. Chuvpilo, M. Zimmer, A. Kerstan, J. Glockner, A. Avots, C. Escher, C. Fischer, I. Inashkina, E. Jankevics, F. Berberich-Siebelt, E. Schmitt, E. Serfling, Alternative polyadenylation events contribute to the induction of NF-ATc in effector T cells, Immunity 10 (1999) 261-269.

[21] S. Diehl, C.W. Chow, L. Weiss, A. Palmetsofer, T. Twardzik, L. Rounds, E. Serfling, R.J. Davis, J. Anguita, M. Rincon, Induction of NFATc2 expression by interleukin 6 promotes T helper type 2 differentiation, J. Exp. Med. 196 (2002) 39-49.

[22] E.M. Rainio, J. Sandholm, P.J. Koskinen, Transcriptional activity of NFATc1 is enhanced by the Pim-1 kinase, J. Immunol. 168 (2002) 1524-1527.

[23] M. Rincon, R.A. Flavell, Transcription mediated by NFAT is highly inducible in effector CD4+ T helper 2 (Th2) cells but not in Th1 cells, Mol. Cell. Biol. 17 (1997) 1522-1534.

[24] J.W. Rooney, T. Hoey, L.H. Glimcher, Coordinate and cooperative roles for NF-AT and AP-1 in the regulation of the murine IL-4 gene, Immunity 2 (1995) 473-483.
[25] J.M. Lumsden, J.M. Roberts, N.L. Harris, R.J. Peach, F. Ronchese, Differential requirement for CD80 and CD80/CD86-dependent co-stimulation in the lung immune response to an influenza virus infection, J. Immunol. 164 (2000) 79-85.

[26] K. McCoy, M. Camberis, G.Le Gros, Protective immunity to nematode infection is induced by CTLA-4 blockade, J. Exp. Med. 186 (1997) 183-187.

[27] S.L. Schreiber, M. Matthias, M. Müller, W. Shaffner, Rapid detection of octamer binding proteins with 'mini'-extracts prepared from a small number of cells, Nucleic Acids Res. 17 (1989) 6419.

[28] J.W. Rooney, M.R. Hodge, P.G. McCaffrey, A. Rao, L.H. Glimcher, A common factor regulates both Th1- and Th2-specific cytokine gene expression, EMBO J. 13 (1994) 625-633.

[29] M.R. Montminy, M. Bilezikjian, Binding of nuclear protein to the cyclic-AMP response element of the somatostatin gene, Nature 328 (1987) 175-178.

[30] M.C. Eichelberger, M.L. Wang, W. Allan, R.G. Webster, P.C. Doherty, Influenza virus RNA in the lung and lymphoid tissue of immunologically intact and CD4-depleted mice, J. Gen. Virol. 72 (1991) 16951698.

[31] J.A. Owen, L. Dudzik, L. Klein, D.R. Dorer, The kinetics of generation of influenza-specific cytotoxic T-lymphocyte precursor cells, Cell. Immunol. 111 (1988) 247-252.

[32] K.J. Flynn, G.T. Belz, J.D. Altman, R. Ahmed, D.L. Woodland, P.C. Doherty, Virus-specific CD8+ T cells in primary and secondary influenza pneumonia, Immunity 8 (1998) 683-691.

[33] S. Sarawar, M. Sangster, R.L. Coffman, P.C. Doherty, Administration of anti-IFN-gamma antibody to beta 2-microglobulin-deficient mice delays influenza clearance but does not switch the response to a helper cell 2 phenotype, J. Immunol. 153 (1994) 1246-1253.

[34] N. Baumgarth, A. Kelso, In vivo blockade of gamma interferon affects the influenza virus-induced humoral and the local cellular immune response in lung tissue, J. Virol. 70 (1996) 4411-4418.

[35] C. Huang, P. Mattjus, W.Y. Ma, M. Rincon, N.Y. Chen, R.E. Brown, Z. Dong, Involvement of nuclear factor of activated T cells activation in UV response. Evidence from cell culture and transgenic mice, J. Biol. Chem. 275 (2000) 9143-9149.

[36] A.S. Stevemsom, M.F. Gomez, D.C. Hill-Eubanks, M.T. Nelson, NFAT4 movement in native smooth muscle, J. Biol. Chem. 276 (2001) $15018-15024$

[37] J.F. Urban, K.B. Madden, A. Svetic, A. Cheever, P.P. Trotta, W.C. Gause, I.M. Katona, F.D. Finkelman, The importance of Th2 cytokines in protective immunity to nematodes, Immunol. Rev. 127 (1992) 205-220.

[38] N.L. Harris, R.J. Peach, F. Ronchese, CTLA4-Ig inhibits optimal T helper 2 cell development but not protective immunity or memory response to Nippostrongylus brasiliensis, Eur. J. Immunol. 29 (1999) 311-316.

[39] N.L. Harris, V. Watt, F. Ronchese, G. Le Gros, Differential T cell function and fate in lymph node and nonlymphoid tissues, J. Exp. Med. 195 (2002) 317-326. 